\title{
A Stepwise Approach to Inbound Passenger Management at a COVID-19 Temporary Medical Observation Site
}

Yongfang Zhang, ',* Di Wang, 2,* Weiqun Liu, ' Yin Zhang, ${ }^{3}$ Lina Zhou, ${ }^{4}$ Hanhuan Yuan, ${ }^{5}$ Jiaming Yao, ${ }^{6}$ Ming Li ${ }^{7}$

'Department of Nursing, Shanghai University of Medicine and Health Sciences Affiliated Zhoupu Hospital, Shanghai, People's Republic of China; ${ }^{2}$ Department of Nursing, Shanghai Jiao Tong University Affiliated Sixth People's Hospital, Shanghai, People's Republic of China; ${ }^{3}$ The Party and Government Office, Shanghai University of Medicine and Health Sciences Affiliated Zhoupu Hospital, Shanghai, People's Republic of China; ${ }^{4}$ Department of Publicity, Shanghai University of Medicine and Health Sciences Affiliated Zhoupu Hospital, Shanghai, People's Republic of China; ${ }^{5}$ Infectious Disease Control and Disinfection Management Section, Shanghai Pudong New Area Center for Disease Control and Prevention, Fudan University Pudong Institute of Preventive Medicine, Shanghai, People's Republic of China; ${ }^{6}$ Columbia University School of Nursing, New York, NY, USA; ${ }^{7}$ Dean's Office, Shanghai University of Medicine and Health Sciences Affiliated Zhoupu Hospital, Shanghai, People's Republic of China

*These authors contributed equally to this work

Correspondence: Ming $\mathrm{Li}$

Shanghai University of Medicine and

Health Sciences Affiliated Zhoupu

Hospital, 1500 W Zhouyuan Road,

Pudong New Area, Shanghai, 201318,

People's Republic of China

Tel +862168135590 Ext. 600I

Fax +86 21-68I357I5

Email liming1070@।26.com

Jiaming Yao

Columbia University School of Nursing, 560

W 168th Street, New York, NY, 10032, USA

$\mathrm{Tel}+\mathrm{I}-646339$ I I 88

Email jy2349@cumc.columbia.edu
Objective: To implement epidemic prevention among entry personnel, Shanghai has launched a number of Medical Observation Sites (Observation Site) for which high expectations are set. Ours is one such Observation Site.

Methods: As part of a novel project, we did not have any previous experience to use as reference. Despite some challenges, we achieved satisfactory outcomes by establishing a stepwise approach to inbound passenger management, including the division of the working area of the Observation Site, dynamic management of the rooms, closed-loop management of the isolated personnel.

Results: As of May 14, 2020, a total of 42 Observation Sites were operational in Pudong New Area. The following are the detailed descriptions of our Temporary Medical Observation Site set up and work flow.

Conclusion: Early screening of inbound passengers as well as prompt and dynamic management of information about passengers' close contacts play an active role in preventing an influx of cases. As a pilot program, we have a model that is effective despite some limitations.

Keywords: COVID-19, observation, pandemic, screening, airport, room

\section{Introduction}

In December 2019, the Centers for Disease Control and Prevention in Wuhan, a city in China's Hubei Province, announced the detection of a cluster of cases of pneumonia with unknown cause. ${ }^{1}$ Since then, the causative organism, SARS-Cov-2, has been identified, and its main route of transmission has been shown to be through respiratory droplets and close contact. ${ }^{2}$ Since the outbreak of the coronavirus 2019 (COVID-19) disease, the Chinese government has urged local medical and health institutions to fully participate in the prevention and control of the epidemic. In March 2020, during a press conference held by the Joint Prevention and Control Mechanism of the State Council, it was announced that the epidemic peak in China had passed, the number of new cases was decreasing, and the epidemic remained at a relatively low level. ${ }^{3}$ However, the outbreak had started to spread and become a global pandemic. Because of the increasing number of cases abroad, many overseas students, foreigners, and Chinese nationals living abroad are returning to China. ${ }^{4}$

To prevent and control COVID-19, some countries or regions have adopted entry control measures. The US has temporarily banned entry of certain groups of 
people, mandated 14-day quarantine, and closed the U.S.Mexico border; Australia prohibits entry to all nonAustralian citizens and non-permanent residents; Japan suspended entry of foreigners from December 28, 2020 to January 2021. ${ }^{5}$ The Shanghai COVID -19 Epidemic Prevention and Control Center announced through a news release on March 27 that starting from March 28, 2020, all persons entering Shanghai would be required to be isolated in a Medical Observation Site (Observation Site) for 14 days. ${ }^{6}$ Exceptions were made for passengers in six special categories: aged above 65, minor, pregnant, maternal, disabled, families who require care for older relatives and children, and individuals with underlying diseases who would not fare well in concentrated isolation. If the nuclear acid test (NAT) is negative and the living environment conforms to home quarantine standards, home quarantine can be implemented after the case is thoroughly evaluated and approved. $^{5}$

The epidemic management of inbound passengers has become an extremely critical element in the prevention and control of COVID-19. To implement epidemic prevention among the entry personnel, Shanghai has launched a number of Observation Sites for which high expectations are set. Ours is one such Observation Site. As part of a novel project, we did not have any previous experience to use as reference, and we faced many challenges such as the high mobility of inbound passengers, the variability of their personal needs, and uncertainty of the risk of crossinfection. However, we achieved satisfactory outcomes by establishing a stepwise approach to inbound passenger management, which is described in further detail below and is the first description of a successful implementation.

Pudong New Area is a district under the jurisdiction of Shanghai, where Shanghai Pudong International Airport is located. Pudong International Airport connects 297 air hubs for 47 countries and regions in the world. ${ }^{7}$ It is one of the main domestic ports of entry, which makes it crucial for epidemic management. To enhance the entry process management of inbound personnel, multiple Observation Sites were set up, including our site. Our service population comprises six special categories of passengers who enter Shanghai from the Pudong International Airport and require home quarantine.

\section{Methods}

Our Observation Site is in a hotel temporarily requisitioned by the district government. It is close to Pudong Airport and distanced from residential areas, and it has more than 300 single rooms. Our team comprises staff from medical, CDC, sanitary supervision, customer service, administration, and other divisions. The medical staff is responsible for performing health assessments, medical observations, and diagnoses, and for offering treatment for inbound passengers' acute diseases. The CDC staff is responsible for directing disinfection, isolation, personal protection, and information reporting. The sanitary supervision staff is responsible for medical material management, medical waste disposal, safety supervision and guidance, etc. The customer service division is responsible for room service, and the administration is responsible for the Observation Site management. All staff members are experienced in the specific field to which they are assigned and receive additional training prior to beginning their work.

After an inbound passenger enters the Pudong International Airport, SARS-Cov-2 NAT is completed at the customs counter. Passengers who belong to one or more of the six special categories fill out the home isolation application form at the airport and specify the reasons they need home isolation. The airport reception team reviews the case, and passengers deemed to be appropriate for home quarantine are transported to our Observation Site using designated transportation (see Figure 1). Upon arrival, each passenger's temperature is taken, and if it is found to be $37.3^{\circ} \mathrm{C}$ and above, that passenger is evaluated by a physician and sent to the designated fever clinic for further investigation. If their temperature is lower than $37.3^{\circ} \mathrm{C}$, the check-in process is initiated.

Subsequently the medical staff conduct a preliminary health assessment on the passenger and provide recommendations on whether he or she falls into one of the six special categories based on the assessment and other medical information, such as medicines taken and recent medical records provided by the passenger. If any inbound passenger feels ill or presents with any symptoms, medical management is provided immediately and, if necessary, that passenger is sent to the nearest designated hospital. By scanning the Pudong New Area Joint Prevention and Control Information Platform QR code, the inbound passenger can register with personal information. The nursing staff then facilitate the check-in process and verify the name, gender, ID number, home address, and contact phone number on the Information Platform. After verification is completed, the room service staff take the passenger to the assigned room to rest and wait for the NAT result.

While waiting for NAT results, community workers check the passenger's home quarantine conditions based on the information provided by the Joint Prevention and Control 


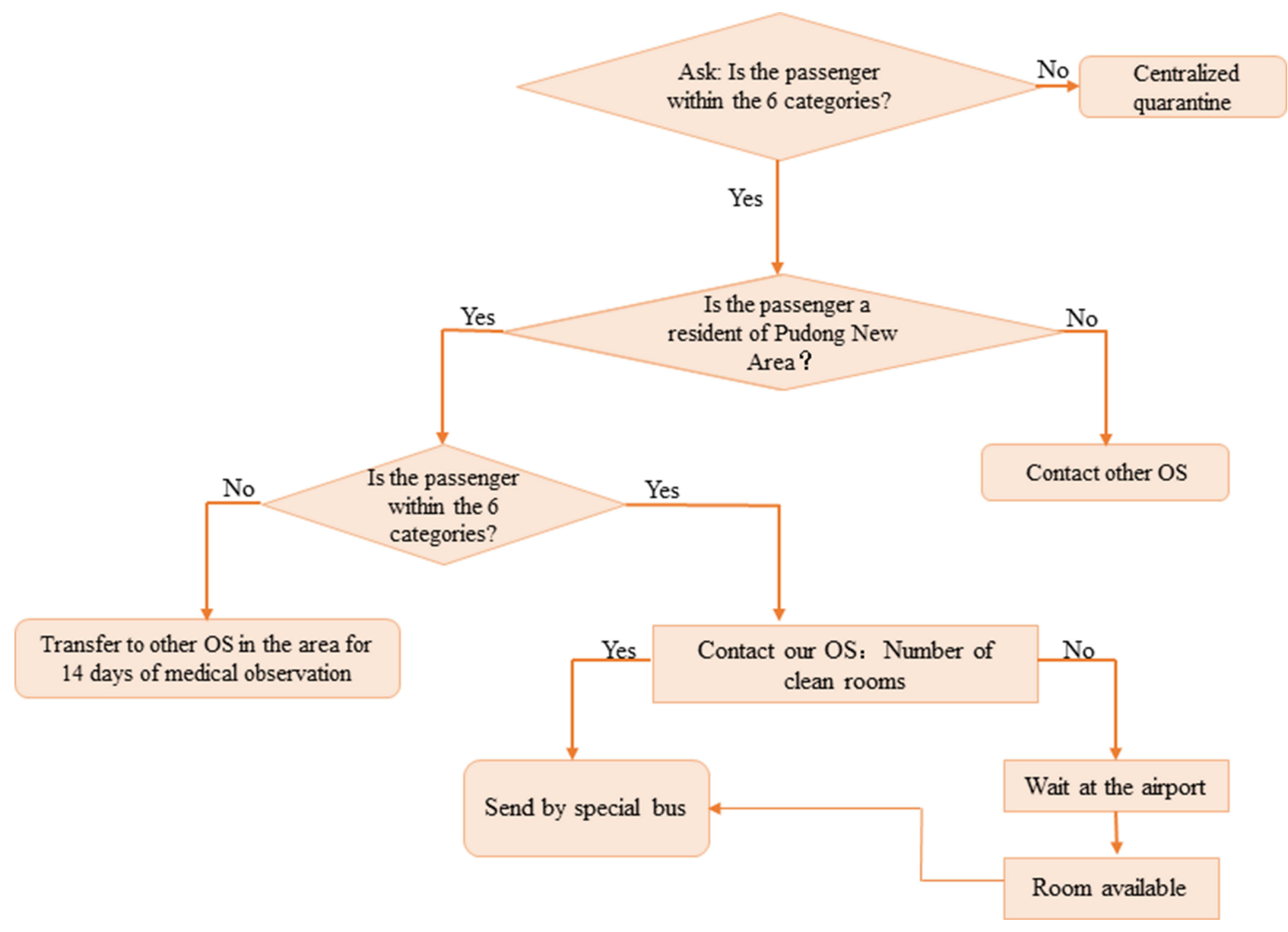

Figure I Inbound passenger flow chart. Abbreviation: OS, Observation Site.

Information Platform. According to the requirement of the Shanghai Health Commission, those who need to be isolated cannot live in the same household with others who do not, in order to avoid cross infection between family members. ${ }^{8}$ The NAT takes about six hours to reveal the results.

Passengers with negative NAT results are sent to their homes or a centralized isolation area by designated transportation, depending on whether their home isolation conditions meet the requirements. Those with positive results are sent to a designated hospital for further testing by emergency center ambulances that are equipped with negative pressure. If the epidemiological analysis indicates, close contacts are also transferred to the designated medical observation sites by negative pressure ambulances for observation (see Figure 2). According to the COVID-19 epidemic prevention and control standards, the room in which each inbound passenger stayed must be professionally disinfected and sealed for at least one hour before sanitary cleaning. These activities must be performed prior to the room being used by another person. The Observation Site can receive and transfer more than 200 passengers every day.

\section{The Observation Site Layout and Features}

Two working areas are set up at the Observation Site: the medical observation area and the office area. The medical observation area is the entire hotel building, with a lobby and more than 300 individual rooms, and is considered a contaminated area. CDC staff are responsible for determination of contaminated area versus office area. The lobby is staffed with medical personnel. The physicians complete the health and home isolation assessments for the inbound passengers in the lobby. The nursing staff checks and verifies the personal information of the passengers, arranges accommodation, provides safety information as well as food and drink, and assists with personal needs. 


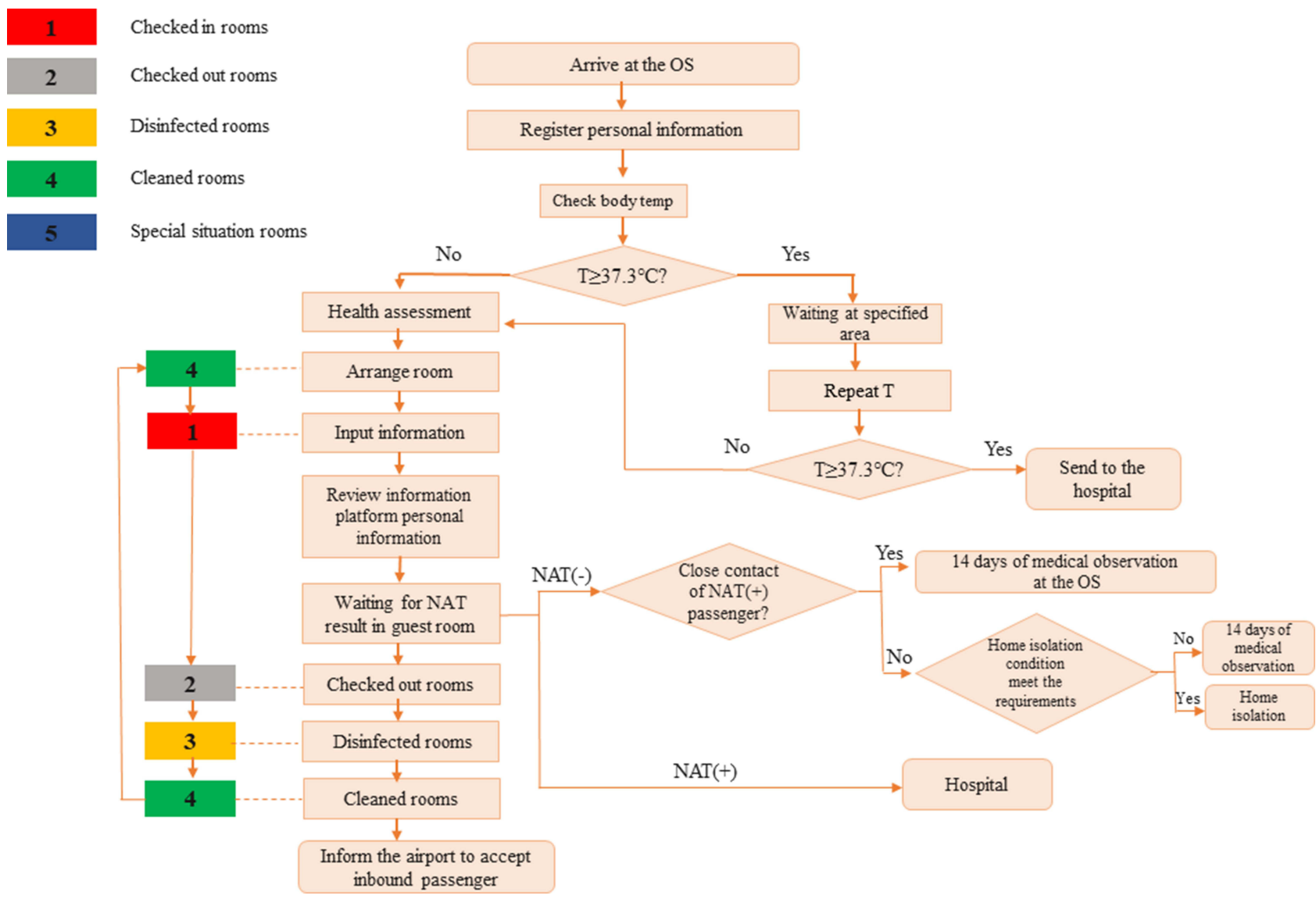

Figure 2 Observation Site operation flow chart.

Abbreviations: OS, Observation Site; NAT, Nuclear Acid Test; T, temperature.

They also arrange for customer service to take passengers to their rooms. The doors are closed and passengers are discouraged from leaving the room to avoid crossinfection. The office area is a multi-division joint office located in a different building from the hotel. It is the central hub of the Observation Site and is considered a clean area.

Since the two working areas are in different locations, it is inconvenient for interactions to take place between the staff from the clean and contaminated areas. There are also other challenges: the Observation Site has a large flow of inbound passengers who arrive at different times; in addition, guest rooms are scattered across a large area. In order to overcome these challenges, we need to have the following information:

The number and distribution of clean rooms: the office staff are then able to inform the airport authorities to divert the corresponding number of inbound passengers to the Observation Site based on the number of the rooms and whether the lobby staff will be able to arrange for the arrival of inbound passengers according to the room distribution and record the relevant information about the occupants in the assigned rooms.

Information about checked-in inbound passengers: the staff of the medical observation area perform medical observations, solve the needs of inbound passengers, and provide appropriate services according to this information. The staff in the office area meet personalized dietary needs, such as halal, an iodine-free diet, or diets recommended for guests with cardiac, renal, and diabetic issues according to their medical history. They contact the community to verify the home isolation conditions of the inbound passengers, and implement the transfer plan after receiving NAT results from customs authorities.

Check-out information: the disinfection staff disinfects the guest rooms according to the check-out information.

Information about the rooms already disinfected: The housekeeper completes the cleaning of the rooms according to the specific information about the rooms already disinfected. To establish a real-time connection between the areas above, dynamic information about the rooms 
needs to be updated promptly, which demands seamless management.

During the early operations of the Observation Site, there was no established information system to support the information sharing between the medical observation and office areas. We used WPS software, supported by shared document system, to combine the shared documents and the transfer slips of room information through the information flow design, and we updated the room status in real time so that the number of available rooms, room status, and the information about the occupants could be shared.

\section{Dynamic Management of Room Information \\ Shared Documents}

Kingsoft Document is a document authoring tool that can be edited by multiple users at the same time. ${ }^{9}$ We designed the document as a WPS spreadsheet that noted the room number, room status, name of the person checked in, ID number, check-in date, remarks column, and so on. After the form design was completed, the sharing link was created using Kingsoft Document. Access and editing authorization were granted to limited users to ensure data security. The sharing link was then sent to staff mobile phones in the two areas via WeChat, a cross-platform communication tool that supports single and multiple user participation through which members can send audio, image, and video files as well as text through the mobile network. The authorized staff can access the link online through the WeChat computer version to open the shared document to edit, change the status of the room, enter information about the inbound passengers in the corresponding room, and note the guests' dietary needs, allergies, special health conditions, and other needs in the remarks column. The office information assistant only needs to open the shared document to access the information about the guests in the rooms in real time.

\section{Room Status}

To prevent cross-infection, even if a passenger only stays in a room for a short period, disinfection and sanitation procedures are still strictly followed. There are five different guest room statuses in the medical observation area:

(1) occupied

(2) checked out

(3) disinfected

(4) cleaned

(5) others (maintenance, was occupied by guest with positive NAT, etc.).

Room status formed a closed loop from check-in to check-out, disinfection, cleaning, and check-in, which promoted the efficiency of the check-in process and maximized the utilization of the rooms. To make the dynamic status of the room clearer and more visible, we used different colors to represent different statuses in the shared document, with red, yellow, green, grey, and blue implying checked in, sterilized, cleaned, checked out, and other, respectively. Each color was associated with a particular number, and the staff changed the filling color by entering numbers in the shared document corresponding to the room status (see Figures 3 and 4).

\section{Transfer Slip of Room Information}

We designed a room information transfer slip (spreadsheet, see Figure 5) to record the corresponding room

\begin{tabular}{|c|c|c|c|c|c|c|c|}
\hline Room number & Room type & room status & Name of check-in guest & Number of guests & Check-in date & Certificate number & Note \\
\hline 8201 & $1.2 \mathrm{~m}$ bed & 1 & & & & \\
\hline 8202 & $1.8 \mathrm{~m}$ bed & 2 & & & & \\
\hline 8203 & $1.2 \mathrm{~m}$ bed & 3 & & & & \\
\hline 8205 & $1.2 \mathrm{~m}$ bed & 4 & & & & \\
\hline 8206 & $1.8 \mathrm{~m}$ bed & 5 & & & & \\
\hline
\end{tabular}

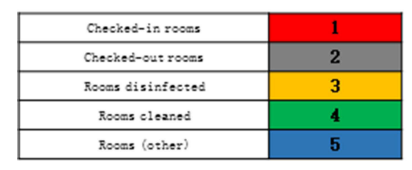

Figure 3 Room information sample. 


\begin{tabular}{|c|c|c|c|c|c|c|c|c|c|c|c|c|}
\hline F10or & Open rooms & $\begin{array}{c}\text { Checked-in } \\
\text { rooms }\end{array}$ & $\begin{array}{c}\text { Guests } \\
\text { already } \\
\text { checked in }\end{array}$ & Clean rooms & $\begin{array}{c}\text { Disinfected } \\
\text { roms }\end{array}$ & $\begin{array}{c}\text { Checked-out } \\
\text { roons }\end{array}$ & $\begin{array}{c}\text { Rooms in } \\
\text { special cases }\end{array}$ & $\begin{array}{c}\text { Guests } \\
\text { staying } \\
\text { longer than } \\
24 \text { hours }\end{array}$ \\
\hline Second floor & 5 & 1 & 0 & 1 & 1 & 1 & 1 & 0 & 0 & 0 & 0 & 1 \\
\hline Total nusber & $\mathbf{5}$ & $\mathbf{1}$ & $\mathbf{0}$ & $\mathbf{1}$ & $\mathbf{1}$ & $\mathbf{1}$ & $\mathbf{1}$ & $\mathbf{0}$ & $\mathbf{0}$ & $\mathbf{0}$ & $\mathbf{0}$ & $\mathbf{1}$ \\
\hline
\end{tabular}

Figure 4 Floor information overview sample.

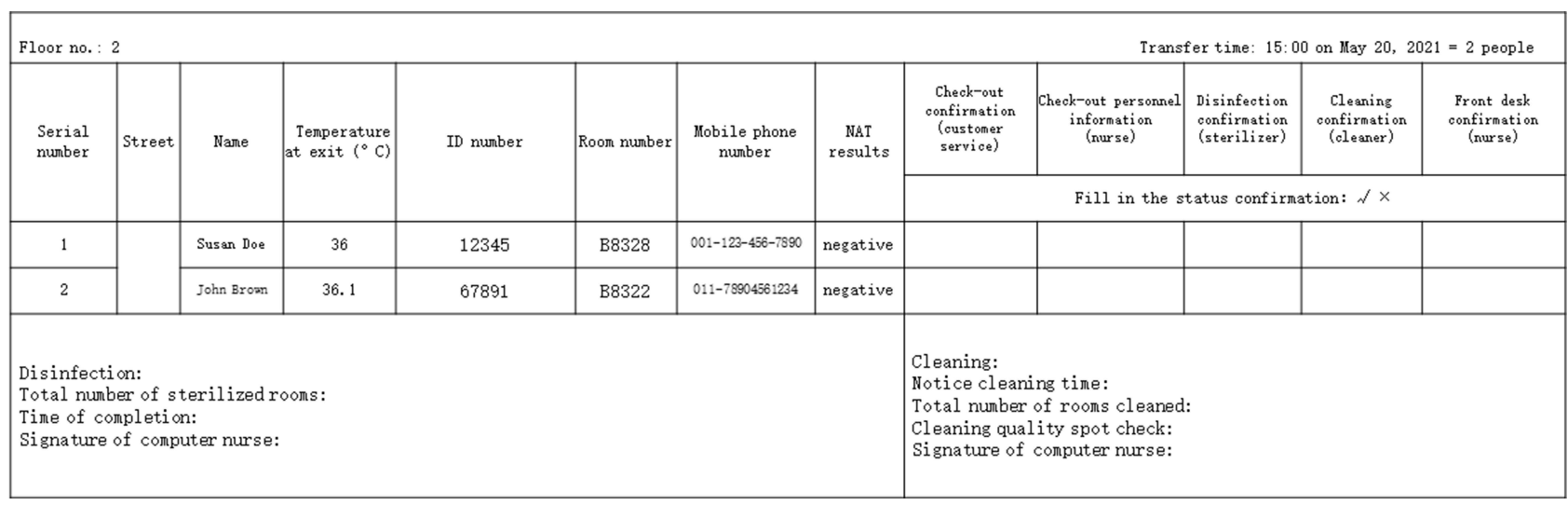

Figure 5 Room information transfer slip sample.

passenger's information and room status, aiming to guide the staff through the process and update the status of the room in real time. The transfer slip includes not only the street name but also the check-out passenger's name, temperature, ID number, room number, mobile phone number, and NAT result, as well as confirmation of check-out, disinfection, cleaning, reception, and front desk.

After the NAT result and transfer notification of the inbound passenger are received, the information officer will complete the electronic transfer slip, contact the corresponding transfer personnel through the telephone regarding pick up time and attention-worthy points. Simultaneously, the spreadsheet will be shared via WeChat with the staff in the medical observation area who will print the transfer slip. The transfer slip is circulated amongst the customer service personnel, nurses, sterilizers, cleaners, and front desk staff, each of whom adds a " $\sqrt{ }$ " mark accordingly for the completed project. The front desk nurse is responsible for changing the room status in the document. Through this process, an effective association between the transfer slip and the shared documents is achieved, and the accuracy of real-time room status in the shared document is verified.

\section{Implementation of Dynamic Management of Guest Rooms \\ Information Sharing}

Dynamic management of guest room information made real-time information sharing possible amongst the medical observation and office areas, thus reducing the occurrence of errors by minimizing miscommunication and promoting work efficiency. External connections were also accelerated by this process, which played a positive role in the quick diversion of inbound flow from the airport.

\section{Color Code Management}

Color code management refers to the application of color, using its marking function to improve the efficacy of management. Using different colors made the room status more recognizable for the staff. Available rooms can be identified easily by searching for green "already cleaned" rooms, which not only simplifies the searching process but also reduces visual fatigue. 


\section{Circulating Single Closed-Loop Management}

Taking into consideration the physical and mental exhaustion of the passengers and the large flow of people at the Observation Site, a quick completion of check-ins was necessary so that the passengers could be provided meals, have their needs met, be informed of their test results, and have transfers arranged in a timely manner. Through dynamic closed-loop management, we conveyed the number of rooms available accurately, carried out disinfection and cleaning work efficiently, and safeguarded the wellbeing of the passengers. This system also simplified the cumbersome process and minimized the occurrence of errors, which helped reduce the risk of cross-infection.

\section{Conclusion}

To fight the COVID-19 outbreak, the current focus of the nation's work is to accelerate vaccination, prevent internal outbreak, and control cases from overseas. According to government published information: As of May 14, 2020, a total of 42 Observation Sites were operational in Pudong New Area, and 89,062 people had been quarantined at home. ${ }^{10}$ As of the writing of this article, China had approximately 6865 total imported cases. ${ }^{11}$ Pudong Airport has the highest volume in inbound passenger SARS-Cov-2 NAT sampling amongst all ports nationwide, with average 2500 sample collections at the customs counter, and maximum 3900 a day. Once an inbound passenger completes SARS-Cov-2 NAT, he/she is transferred to an Observation Site using designated transportation if deemed to be appropriate for home quarantine by the airport reception team. ${ }^{12}$ Based on our experience, early screening of inbound passengers as well as prompt and dynamic management of information about passengers' close contacts are crucial factors in preventing an influx of cases.

One limitation of this paper is, due to certain policies and regulations, we are unable to provide detailed statistical data of the Observation Site. There are also some systematic limitations in data management, development, and utilization, all of which are confined to the operating platform condition, such as that historical information cannot be retrieved, that we are unable to extract statistics on passengers' personal information, and that passengers' information is still manually entered. However, while COVID-19 cases continue to grow globally, this management strategy can still serve an important role in COVID 19 prevention in countries with low vaccination rates.

\section{Implications for Policy \& Practice}

- Our experience with current system clearly suggests that early screening of inbound passengers as well as prompt and dynamic management of information about passengers' close contacts play an active role in preventing an influx of cases. This model is effective despite some limitations.

- Since COVID-19 has become a global pandemic, many countries are facing the challenges of attempting to prevent an influx of cases. Our program can be considered a pilot program to provide reference and some guidance for practices in other areas in China and even across the world.

- Future utilization of a more powerful and robust operating platform will allow all Observation Sites to connect with each other, as will the Pudong New Area Joint Prevention and Control Information Platform, which will form a large database to meet future COVID-19 prevention and research needs. We urge stakeholders to support such programs by providing more funding and resources.

\section{Acknowledgments}

All persons who have made substantial contributions to the work reported in the manuscript (eg, technical help, writing and editing assistance, general support), but who do not meet the criteria for authorship, are named in the Acknowledgements and have given us their written permission to be named. If we have not included an Acknowledgements, then that indicates that we have not received substantial contributions from non-authors. Yongfang Zhang and Di Wang are co-first authors for this study. Jiaming Yao and Ming $\mathrm{Li}$ are cocorrespondence authors for this study.

\section{Author Contributions}

All authors made a significant contribution to the work reported, whether that is in the conception, study design, execution, acquisition of data, analysis and interpretation, or in all these areas; took part in drafting, revising or critically reviewing the article; gave final approval of the version to be published; have agreed on the journal to which the article has been submitted; and agree to be accountable for all aspects of the work.

\section{Funding}

This study was funded by the Pudong New Area Science and Technology Committee (PKJ2019-Y38). 


\section{Disclosure}

The authors reports no conflicts of interest in this work.

\section{References}

1. The CCTV. China has released information on COVID-19 and promoted international cooperation in epidemic prevention and control; 2021. Available from: http://news.cctv.com/2020/04/06/ ARTINo7ZDjMPratbdqaNIt0j200406.shtml. Accessed April 6, 2021.

2. Huang C, Wang Y, Li X, et al. Clinical features of patients infected with 2019 novel coronavirus in Wuhan, China. Lancet. 2020;395 (10223):497-506. doi:10.1016/S0140-6736(20)30183-5

3. The State Council the People's Republic of China. The press conference of the joint prevention and control mechanism of the state council; 2021. Available from: http://www.gov.cn/xinwen/202003/12/ content_5490604.htm. Accessed April 6, 2021.

4. The Centers for Disease Control and Prevention. The national health commission deployed COVID-19 epidemic prevention and control work; 2021. Available from: http://www.nhc.gov.cn/jkj/s7915v/ 202105/bcd7f8209f744139b141685902bc84c3.shtml. Accessed July 14, 2021.

5. National Immigration Administration. Enquiry on temporary immigration control measures to country of entry; 2021. Available from: https://www.nia.gov.cn/n741435/n907688/n1234186/n1248051/index. html. Accessed July 14, 2021.
6. Shanghai Health Commission. A press release; 2020. Available from: http:// wsjkw.sh.gov.cn/xwfb/20200328/607c2936f40b4750998e51f51d025947. html. Accessed April 6, 2021.

7. Civil aviation administration of China. From the world's 37th to the world's second China became a major air transport country; 2020. Available from: http://www.caac.gov.cn/XWZX/MHYW/ 200812/t20081204_12164.html. Accessed April 6, 2021.

8. Shanghai Health Commission. A press release. 2020 Mar 17 [cited May 20, 2020]. Available from: http://wsjkw.sh.gov.cn/ xwfb/20200317/678c4eb3ab87439c8a391f16c889cf6b.html. Accessed April 6, 2021.

9. Kingsoft document. Multi-person real-time collaboration of online office; 2020. Available from: https://www.kdocs.cn/welcome\#home. Accessed Apr 6, 2021.

10. Information Office of Shanghai Municipal. On May 14, Shanghai situation of prevention and control of COVID - 19; 2020 May 14. Available from: http://www.shio.gov.cn/sh/xwb/n790/n792/n1114/ n1123/u1ai24694.html. Accessed April 6, 2021.

11. National Health Commission of China. As of July 13 COVID - 19 epidemic updates; 2021. Available from: http://www.nhc.gov.cn/xcs/ yqtb/202107/923f961757074387a2d11647e5b6292d.shtml. Accessed July 14, 2021.

12. Shanghai Customs People's Republic of China. Pudong Airport's sampling volume ranks first among airports in the country; 2021. Available from: http://shanghai.customs.gov.cn/shanghai_cus toms/423446/423447/3742754/index.html. Accessed July 15, 2021.
Risk Management and Healthcare Policy

\section{Publish your work in this journal}

Risk Management and Healthcare Policy is an international, peerreviewed, open access journal focusing on all aspects of public health, policy, and preventative measures to promote good health and improve morbidity and mortality in the population. The journal welcomes submitted papers covering original research, basic science, clinical \& epidemiological studies, reviews and evaluations,

\section{Dovepress}

guidelines, expert opinion and commentary, case reports and extended reports. The manuscript management system is completely online and includes a very quick and fair peer-review system, which is all easy to use. Visit http://www.dovepress.com/testimonials.php to read real quotes from published authors. 